\title{
Comparison of Various Means of Evaluating Molecular Electrostatic Potentials for Noncovalent Interactions
}

\author{
Steve Scheiner $\mathbb{1}$
}

The various heterodimers formed by a series of Lewis acids with $\mathrm{NH}_{3}$ as Lewis base are identified. Lewis acids include those that can form chalcogen ( $\mathrm{HSF}$ and $\mathrm{HSBr}$ ), pnicogen $\left(\mathrm{H}_{2} \mathrm{PF}\right.$ and $\left.\mathrm{H}_{2} \mathrm{PBr}\right)$, and tetrel $\left(\mathrm{H}_{3} \mathrm{SiF}\right.$ and $\left.\mathrm{H}_{3} \mathrm{SiBr}\right)$ bonds, as well as $\mathrm{H}$-bonds and halogen bonds. The molecular electrostatic potential (MEP) of each Lewis acid is considered in a number of ways. Pictorial versions show broad regions of positive and negative MEP, on surfaces that vary with respect to either the value of the chosen isopotential, or their distance from the nuclei. Specific points are identified where the MEP reaches a maximum on a particular isodensity surface $\left(V_{s, \max }\right)$. The locations and values of $V_{s, \max }$ were evaluated on different isodensity surfaces, and compared to the stabilities of the various equilibrium geometries. As the chosen isodensity is decreased, and the MEP maxima drift away from the molecule, some points maintain their angular positions with respect to the molecule, whereas others undergo a reorientation. The lowering isodensity also causes some of the maxima to disappear. In general, there is a fairly good correlation between the energetic ordering of the equilibrium structures and the values of $V_{s, \max }$. A number of possible Lewis acid sites on the heteroaromatic imidazole ring were also considered and presents some cautions about application of $V_{s, \max }$ as the principal criterion for predicting equilibrium geometries. (c) 2017 Wiley Periodicals, Inc.

DOI: $10.1002 / j c c .25085$

\section{Introduction}

Noncovalent bonds by their nature involve a longer separation between participating atoms than do their covalent counterparts. ${ }^{[1-3]}$ Moreover, one can clearly identify a pair of entities, clusters of atoms, which interact with one another. Using the $\mathrm{H}$-bond $(\mathrm{HB})$ as a convenient example, the proton donor and acceptor units are typically full-fledged molecules, connected together by an attractive force, but still clearly identifiable as separate units. ${ }^{[4-8]}$ As such, there are certain components of the intermolecular interaction that have simple physical interpretations. The most obvious of these is the Coulombic attraction between the charge distributions of the two constituent molecules. More specifically, each molecule can be thought of as a set of nuclear point charges, floating in a sea of electron density. The latter is not uniform, but rather lumpy, with pockets of higher density amid lower-density regions. The molecular electrostatic potential (MEP) can then be straightforwardly computed by Coulomb's Law, taking account of both the positive nuclei and integrating over the negative electron density. ${ }^{[9,10]}$

As a long-range interaction, electrostatic attraction ought to be the first force felt by the two molecules as they approach one another. One can thus envision a scenario wherein these Coulombic forces guide the two molecules as they dock with one another into their equilibrium geometry within the heterodimer. Of course, other forces are present as well, ${ }^{[1-17]}$ most notably the polarization of each molecule's charge cloud by the other, and a dispersive attraction. There is also a steric repulsion that prevents the two molecules from fully merging into a single entity. But given its long-range character, as well as its strength, it is the electrostatic force that has been very commonly used, at least as a first iteration, to understand the geometry of the equilibrium heterodimer structure, as well as the magnitude of the binding energy. It has been said for example, that $\mathrm{H}$-bonding is primarily an electrostatic phenomenon. Although the contribution of this force to an HB is indisputably very substantial, arguments continue about the relative magnitude and effects of other components of this interaction.

It is not only HBs that have been examined in the context of electrostatic attraction as a possible driving force but also a number of closely related interactions. The replacement of the bridging $\mathrm{H}$ atom by a halogen leads to the now well-known halogen bond, ${ }^{[18-22]}$ which has striking similarities with the $\mathrm{HB}$, despite the much larger shared atom. Halogen and HBs have comparable strength, strive toward linearity, can be decomposed into components of similar magnitude, and manifest the same sort of $n \rightarrow \sigma^{*}$ charge transfer phenomenon. The same can be said of the chalcogen, pnicogen, and tetrel bonds, in which the halogen is replaced by an atom of the eponymous family of the periodic table. ${ }^{[23-36]}$

Gadre's group has been heavily involved in providing some basic concepts by which the MEP might be interpreted and utilized to best advantage. An early analysis of the theoretical underpinnings of $\mathrm{MEPs}^{[37]}$ showed that while minima can occur, the presence of a true local maximum is not possible, although of course a maximum can occur if one is restricted

\footnotetext{
S. Scheiner

Department of Chemistry and Biochemistry, Utah State University, Logan,

Utah 84322-0300 E-mail: steve.scheiner@usu.edu

(c) 2017 Wiley Periodicals, Inc.
} 
to a particular isodensity surface. This group also found soon thereafter ${ }^{[38]}$ that the same is true when the system in question contains an overall negative charge, but emphasized the rich topographical details that may be gleaned if one studies the MEP in detail, much superior to the features of the electron density. They stressed the value of locating a variety of critical points on the MEP. For example, there will be present between any pair of bonded atoms negative-valued saddle points of $(3,-1)$ type, and frequently $(3,+1)$ as well. Gadre and Bhadane ${ }^{[39]}$ later showed the value of the MEP in examination of $\mathrm{H}$-bonding, in particular a strong correlation between the position of the most negative MEP point and the length of the bond. The utility of the MEP is not limited to bonds, as more recent work $^{[40]}$ has utilized the topography of MEPs to more precisely define the location and extension of lone electron pairs. The authors established criteria based on the value of the eigenvalue at a critical point, and the direction in which the associated eigenvector points.

Following incorporation of the MEP ideas into the chemistry community in a widely read tome, ${ }^{[9]}$ Politzer et al have applied this concept to a wide variety of systems. For example, its correlation with a H-bonding parameter was discussed in 1991, ${ }^{[41]}$ and acidity/basicity ideas several years later, $^{[42]}$ as well as how it might be useful in the design of new materials. ${ }^{[43]}$ The notion was applied soon thereafter to larger biological systems ${ }^{[44]}$ and thence to its incorporation as a stalwart parameter in halogen bonds, ${ }^{[45]}$ later expanded to $\sigma$-hole interactions in general. ${ }^{[46]}$

While a large number of studies ${ }^{[41,44,47-56]}$ have examined the MEP, the electron density, and their relation to the properties of the incipient noncovalent bond, there remain numerous questions about details of its actual implementation. There is little question concerning how to calculate this object, which amounts to a value at all points in space surrounding the molecule in question. Conversely, there are a number of ways in which this field of values can be presented, understood, and used. One can, for example, choose a particular value of the MEP, both positive and negative, and display the locus of points with this particular value. The extent of this isopotential surface in various directions in space can provide insights into how an approaching system might view the molecule. An alternate pictorial description considers a surface of fixed distance from the various nuclei, for example, their van der Waals radii. On this surface, the value of the MEP can be indicated by one of several colors, typically blue for the most positive and red for extreme negative. By matching opposite colors, for example, blue (positive) on one molecule with red (negative) on its partner, one can anticipate the optimal orientations of the two molecules as they approach one another. The latter pictorial analysis can be made more quantitative as follows. Once a particular surface has been chosen, say one of constant electron density, the points on this isodensity surface where the MEP attains extreme values, both maxima and minima, can be located, and the value of the MEP at each such point evaluated. Comparison of these quantities between closely related monomers, for example, chlorosubstituted versus fluorosubstituted, can lead to predictions as to which of the two molecules would tend toward a stronger Coulombic attraction with the oppositely charged portion of the partner molecule's MEP.

No matter the type of analysis, there are a number of issues of arbitrariness. For example, which value of MEP will best allow an isopotential surface to represent the types of interactions that a given molecule might form? As the value chosen becomes smaller, the isopotential surface will extend further and further away from the molecule. If distance from nuclei is taken as the constant parameter for definition of a surface, what distance is optimal. Similarly for isodensity surfaces, which particular density best facilitates prediction and understanding of the types of equilibrium geometries that will be formed by the pair of molecules? As the selected isodensity diminishes, the surface will move outward from the molecule, and the overall potential become less positive. This change will, therefore, affect not only the values of the maxima and minima on the isodensity surface but may also influence their positions with respect to the various atoms.

The data presented here reflect an attempt to describe in some detail how these various representations of the MEP correlate with one another. Which sort of picture is likely to best enable one to understand the various configurations that may be adopted by a given pair of molecules? And within a given framework, say the isodensity surface representation, what is the optimal density to make qualitative, and even quantitative, predictions as to the energetics of equilibrium dimer structures?

\section{Systems and Computational Methods}

$\mathrm{HSF}$ and $\mathrm{HSBr}$ were taken as prototypes of molecules that can engage in chalcogen, as well as $\mathrm{H}$ and halogen bonds. The replacement of $\mathrm{F}$ by $\mathrm{Br}$ is expected to strengthen any halogen bond that might be weak or nonexistent for HSF. This same substitution, and the lesser electronegativity of $\mathrm{Br}$, ought to also weaken the chalcogen bond to perhaps make it more competitive with the other sorts of bonds. $\mathrm{H}_{2} \mathrm{PF}$ and $\mathrm{H}_{2} \mathrm{PBr}$ serve similar functions as pnicogen-bonding molecules, that also have the capability to engage in $\mathrm{H}$ and halogen bonds. The $\mathrm{Si}$ atom of $\mathrm{H}_{3} \mathrm{SiF}$ and $\mathrm{H}_{3} \mathrm{SiBr}$ expands the scope of this work to tetrel bonds. To broaden the discussion, and to compare more than one $\mathrm{H}$-bonding site, the heteroaromatic imidazole ring was also considered as a Lewis acid. $\mathrm{NH}_{3}$ was taken as the universal electron donor, due to the simplicity of its single lone pair, and with no $\pi$ bonds that might complicate the analysis by acting as an alternate donor site.

All calculations were carried out within the framework of the Gaussian-09 ${ }^{[57]}$ set of codes. The aug-cc-pVDZ basis set was applied at the MP2 level. All geometries were fully optimized with no geometrical restrictions. The binding energy, $E_{\mathrm{b}}$, of each complex was evaluated as the difference between the energy of the complex and the sum of the energies of separately optimized monomers. Each binding energy was corrected for basis set superposition error by the counterpoise procedure. ${ }^{[58]}$ Geometries and MEP maps were visualized with the Chemcraft program. ${ }^{[59]}$ Maxima on the isodensity surfaces 


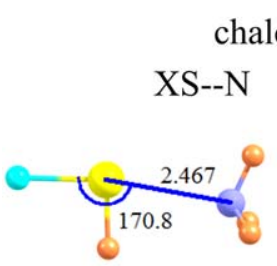

7.92

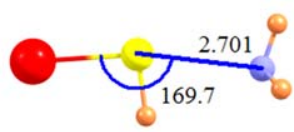

4.72 chalcogen bond

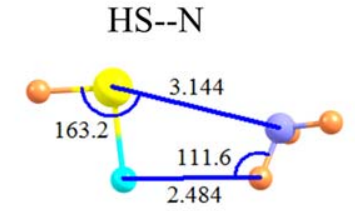

1.98

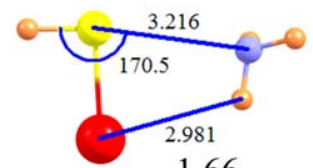

H-bond

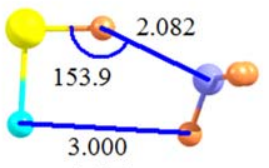

4.77

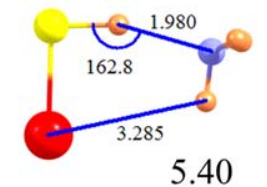

X-bond

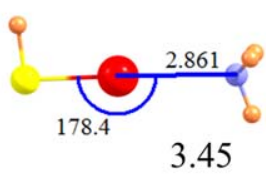

Figure 1. Optimized geometries of HSF (top) and $\mathrm{HSBr}$ (bottom) with $\mathrm{NH}_{3}$. Distances in $\AA$, and angles in degrees, with binding energies in $\mathrm{kcal} / \mathrm{mol}$. [Color figure can be viewed at wileyonlinelibrary.com]

were located and quantified via the Multiwfn program, version 3.3.8. ${ }^{[60]}$

\section{Results}

The optimized geometries found for the various complexes of $\mathrm{HSF}$ with $\mathrm{NH}_{3}$ are exhibited in the top half of Figure 1. The corresponding structures for the $\mathrm{HSBr}$ analogues are presented in the lower half. These structures fall into one of three primary categories. The geometries wherein the $\mathrm{N}$ lone pair of $\mathrm{NH}_{3}$ approaches the $\mathrm{S}$ atom are termed chalcogen bonds. As may be seen on the left side of Figure 1, the $\mathrm{N}$ atom may be situated either opposite the halogen $(\mathrm{XS} \cdots \mathrm{N})$ or the $\mathrm{H}$ atom $(\mathrm{HS} \cdots \mathrm{N})$. The $\mathrm{HBs}$ involve a $\mathrm{SH} \cdot \mathrm{N}$ arrangement and the halogen $(\mathrm{X})$ bonds orient the $\mathrm{N}$ lone pair toward the halogen atom. Note that there is no halogenbonded structure for HSF, which exemplifies the reluctance of $\mathrm{F}$ to engage in such bonds.

The pnicogen and tetrel analogues of $\mathrm{HSF} / \mathrm{HSBr}$ are $\mathrm{H}_{2} \mathrm{PF} /$ $\mathrm{H}_{2} \mathrm{PBr}$ and $\mathrm{H}_{3} \mathrm{SiF} / \mathrm{H}_{3} \mathrm{SiBr}$, respectively; the structures of the minima identified for these molecules with $\mathrm{NH}_{3}$ are displayed in Figures 2 and 3. These configurations fall into the same general categories of noncovalent interactions as HSX. It may be noted that only the $\mathrm{Br}$ derivatives engage in halogen bonds. An $\mathrm{HB}$ only occurs for $\mathrm{H}_{2} \mathrm{PBr}$, but this designation is questionable, with a $\mathrm{PH} \cdot \mathrm{N}$ angle of $143^{\circ}$.

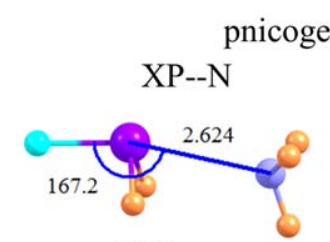

6.18

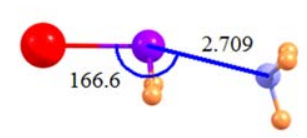

5.03

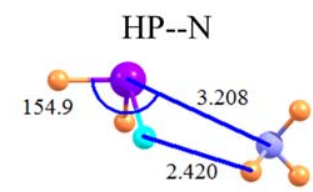

2.54
The first column of Tables 1-3 reports the binding energy of each of these complexes, also displayed as the black numbers in the corresponding figures. In the case of HSF, the FS..N chalcogen bond is the strongest, followed by the $\mathrm{SH} \cdot \mathrm{N} \mathrm{HB}$, and then by the HS..N chalcogen bond. (The latter two configurations may have slightly exaggerated binding energies, due to the presence of a weak angularly distorted $\mathrm{NH} . . \mathrm{F} \mathrm{HB}$ which slightly contaminates the pure $\mathrm{HS} \cdot \cdot \mathrm{N}$ and $\mathrm{SH} \cdot \cdot \mathrm{N}$, respectively.) On changing the $\mathrm{F}$ atom to the larger $\mathrm{Br}$, the XS..N chalcogen bond is weakened a bit, due in large part to the lesser electronegativity of $\mathrm{Br}$. The $\mathrm{Br}$ atom is known to be much more prone than $\mathrm{F}$ to engage in a halogen bond, and does so, as may be seen by the furthest right diagram in Figure 1. The HB structure is the most stable of this quartet, followed by the BrS..N chalcogen bond, then by the halogen bond, and lastly by the weaker HS..N chalcogen bond. Importantly, the order of stability changes when replacing $\mathrm{F}$ by $\mathrm{Br}$. The most stable structure switches from FS. N chalcogen to a $\mathrm{SH} \cdot$. N HB. As a secondary issue, the replacement also allows the formation of a halogen bond, not present for HSF.

\section{Isopotential maps}

The question arises as to how well a simple analysis of the MEP of the pair of molecules would enable a prediction as to these results. One means of examining the MEP is via a three-
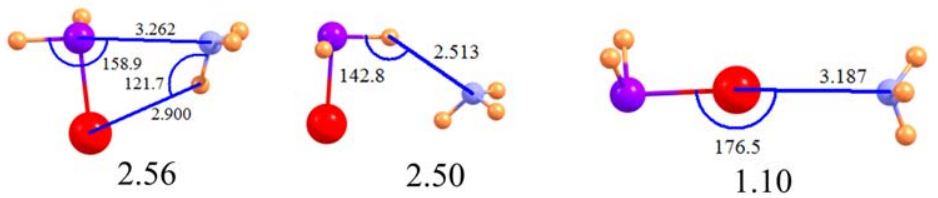

Figure 2. Optimized geometries of $\mathrm{H}_{2} \mathrm{PF}$ (top) and $\mathrm{H}_{2} \mathrm{PBr}$ (bottom) with $\mathrm{NH}_{3}$. Distances in $\AA$, and angles in degrees, with binding energies in kcal/mol. [Color figure can be viewed at wileyonlinelibrary.com] 


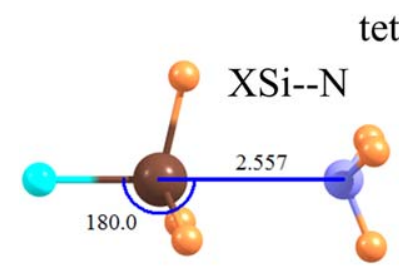

4.94
5.49

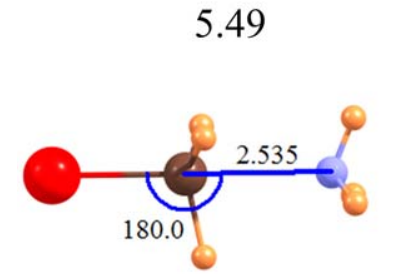

tetrel bond

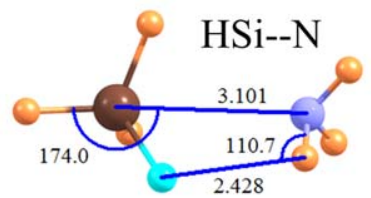

2.90

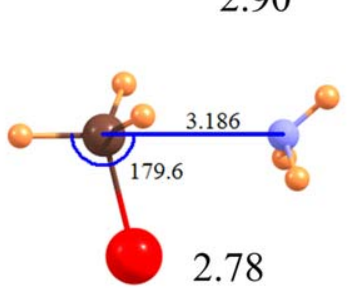

X-bond

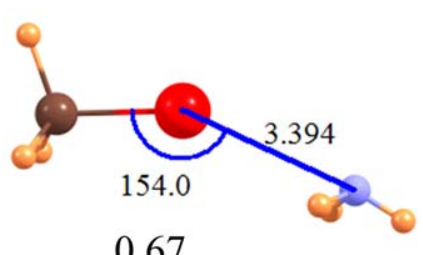

0.67

Figure 3. Optimized geometries of $\mathrm{H}_{3} \mathrm{SiF}$ (top) and $\mathrm{H}_{3} \mathrm{SiBr}$ (bottom) with $\mathrm{NH}_{3}$. Distances in $\AA$, and angles in degrees, with binding energies in kcal/mol. [Color figure can be viewed at wileyonlinelibrary.com]

dimensional picture as in Figure 4. This plot illustrates a surface of constant MEP, an isopotential contour. The blue color represents positive potential, while negative is shown in red. The particular isopotential shown in Figure 4 is \pm 0.015 au, although any value could of course be chosen instead. (For the molecules considered here, larger values of the isopotential tend to miss the red negative regions, whereas smaller isodensity surfaces extend too far away from the molecule.)

How well do these MEP maps predict the equilibrium geometries? Taking HSF as a first example, the blue region extends to the right of the molecule, away from F. The blue surface is rather broad, and would encompass the FS.-N chalcogen bond and the $\mathrm{HB}$ configurations in Figure 1. But there is little grounds for guessing one might be stronger than the other. One could also make the argument that the extension of the blue region directed away from the $\mathrm{H}$ atom might also lead to the expectation of a HS..N chalcogen bond, albeit a weaker one. Note that there is no blue region to the left of the $F$ atom, which is consistent with the lack of a halogen-bonded structure. This observation stands in stark contrast to the blue region to the left of the $\mathrm{Br}$ atom in $\mathrm{HSBr}$, consistent with the identification of a $\mathrm{SBr} \cdot \mathrm{N}$ halogen bond in Figure 1. In fact, the area around this molecule is blue in nearly all directions,

\begin{tabular}{|c|c|c|c|c|c|}
\hline HSF & $\begin{array}{c}E_{\mathrm{b}} \\
(\mathrm{kcal} / \mathrm{mol})\end{array}$ & $\rho=0.01$ & $\rho=0.001$ & $\rho=0.0002$ & $\rho=0.00005$ \\
\hline X-S & 7.92 & 136.44 & 48.63 & 29.37 & 21.52 \\
\hline $\mathrm{H}-\mathrm{S}$ & 1.98 & 81.88 & 17.01 & 5.86 & 2.53 \\
\hline $\mathrm{S}-\mathrm{H}$ & 4.77 & 96.68 & 35.66 & $x$ & $x$ \\
\hline S-X & $x$ & -12.59 & -18.46 & $x$ & $x$ \\
\hline \multicolumn{6}{|l|}{$\mathrm{HSBr}$} \\
\hline$X-S$ & 4.72 & 89.56 & 29.09 & $x$ & $x$ \\
\hline $\mathrm{H}-\mathrm{S}$ & 1.66 & 61.68 & 10.78 & 3.31 & 1.02 \\
\hline S-H & 5.40 & 90.61 & 31.80 & 20.31 & 14.71 \\
\hline$S-X$ & 3.45 & 78.31 & 19.43 & 9.75 & 6.28 \\
\hline
\end{tabular}

particularly large around the $\mathrm{H}$ atom, which comports with the finding of a stable $\mathrm{SH} \cdot \cdot \mathrm{N}$ HB.

Turning next to the pnicogens, the blue region around $\mathrm{H}_{2} \mathrm{PX}$ is particularly extended opposite the $\mathrm{F}$ and $\mathrm{Br}$ atoms, which is fully consistent with the strong XP..N pnicogen bonding structures. There is also a smaller bump opposite the $\mathrm{H}$ atoms, which are predictive of the HP..N pnicogen bonds. One potential weakness of this style of presentation of MEP is related to the very similar patterns around the $\mathrm{H}$ atoms in $\mathrm{H}_{2} \mathrm{PF}$ and $\mathrm{H}_{2} \mathrm{PBr}$. However, it is only the latter molecule that actually engages in a $\mathrm{H}$-bonded structure. As in the case of HSX, it is only for $\mathrm{H}_{2} \mathrm{PBr}$ that there is a blue region to the left of the halogen that facilitates formation of the $\mathrm{PBr} \cdot \mathrm{N}$ bond. The blue region to the right of the $\mathrm{Si}$ atoms have "lumps" that correctly suggest the existence of $\mathrm{XSi} \cdot \cdot \mathrm{N}$ and $\mathrm{HSi} \cdot \mathrm{N}$ tetrel bonds. The isodensity maps again presage the existence of a halogen bond for Br but not for $\mathrm{F}$. Conversely, the lumps that surround the $\mathrm{H}$ atoms do not result in $\mathrm{H}$-bonded geometries for the $\mathrm{Si}$ molecules.

\section{MEP maps at given distance from nuclei}

As an alternative to viewing a surface of constant MEP, one can also consider a surface of a particular distance from nuclei. On such a surface, the MEP will typically have many values,

Table 2. Binding energies of indicated $\mathrm{H}_{2} \mathrm{PX}$ molecules with $\mathrm{NH}_{3}$, and $V_{\mathrm{s}, \max }(\mathrm{kcal} / \mathrm{mol})$ on indicated isopotential surface of monomer, $\rho$ in au.

\begin{tabular}{|c|c|c|c|c|c|}
\hline $\mathrm{H}_{2} \mathrm{PF}$ & $\begin{array}{c}E_{\mathrm{b}} \\
(\mathrm{kcal} / \mathrm{mol})\end{array}$ & $\rho=0.01$ & $\rho=0.001$ & $\rho=0.0002$ & $\rho=0.00005$ \\
\hline$X-P$ & 6.18 & 123.79 & 39.14 & 22.18 & 15.66 \\
\hline $\mathrm{H}-\mathrm{P}$ & 2.54 & 82.94 & 17.24 & $x$ & $x$ \\
\hline $\mathrm{P}-\mathrm{H}$ & $x$ & 50.61 & $x$ & $x$ & $\mathrm{x}$ \\
\hline$P-X$ & $x$ & -13.71 & -18.75 & $x$ & $x$ \\
\hline \multicolumn{6}{|l|}{$\mathrm{H}_{2} \mathrm{PBr}$} \\
\hline$X-P$ & 5.03 & 103.45 & 30.15 & 17.51 & 12.96 \\
\hline $\mathrm{H}-\mathrm{P}$ & 2.56 & 71.20 & 15.02 & $x$ & $x$ \\
\hline $\mathrm{P}-\mathrm{H}$ & 2.50 & 55.53 & $x$ & $x$ & $x$ \\
\hline$P-X$ & 1.10 & 52.56 & 6.94 & 1.14 & -0.56 \\
\hline
\end{tabular}


Table 3. Binding energies of indicated $\mathrm{H}_{3} \mathrm{SiX}$ molecules with $\mathrm{NH}_{3}$, and $V_{\mathrm{s}, \max }(\mathrm{kcal} / \mathrm{mol})$ on indicated isopotential surface of monomer, $\rho$ in au.

\begin{tabular}{|c|c|c|c|c|c|}
\hline $\mathrm{H}_{3} \mathrm{SiF}$ & $\begin{array}{c}E_{\mathrm{b}} \\
(\mathrm{kcal} / \mathrm{mol})\end{array}$ & $\rho=0.01$ & $\rho=0.001$ & $\rho=0.0002$ & $\rho=0.00005$ \\
\hline X-Si & 5.49 & 154.36 & 40.98 & 21.04 & 14.01 \\
\hline $\mathrm{H}-\mathrm{Si}$ & 2.90 & 128.80 & 26.20 & 9.75 & 5.22 \\
\hline $\mathrm{Si}-\mathrm{H}$ & $x$ & 33.70 & $x$ & $x$ & $x$ \\
\hline Si-X & $x$ & $x$ & $x$ & $x$ & $x$ \\
\hline \multicolumn{6}{|l|}{$\mathrm{H}_{3} \mathrm{SiBr}$} \\
\hline X-Si & 4.94 & 142.98 & 36.62 & 18.24 & 12.23 \\
\hline $\mathrm{H}-\mathrm{Si}$ & 2.78 & 111.50 & 21.80 & 8.79 & $x$ \\
\hline $\mathrm{Si}-\mathrm{H}$ & $x$ & 37.30 & $x$ & $x$ & $x$ \\
\hline $\mathrm{Si}-\mathrm{X}$ & 0.67 & 40.68 & 2.83 & -1.28 & -2.28 \\
\hline
\end{tabular}

and of both sign. These domains of positive and negative MEP can be displayed via different colors, for example, blue for most positive, red for most negative, with intermediate regions in yellow or green. Surfaces of this type are displayed in Figure 5 for HSF. Within this general scheme, there are multiple variations. For example, the top half of Figure 5 uses +0.1 and -0.1 au as the extrema of the MEP, whereas the lesser values of $\pm 0.02 \mathrm{au}$ are used in the lower half. Another point at issue regards just how far from the atoms one should evaluate the MEP. The two diagrams on the far left represent a surface corresponding to the van der Waals radii of each of the various atoms. But it is unlikely that an approaching nucleophile will come in this close, so longer distances are of interest as well. The second set of diagrams in Figure 5 correspond to 1.5 times the vdW radii, followed by twice this radius on the farthest right.

There are several patterns that are immediately evident. In the first place, moving from left to right, that is, transitioning outward from the nuclei, causes the overall potential to become less positive, that is, less blue. This result is due to the greater shielding of the nuclei by the larger portion of the electron density encompassed by the larger radii. Because of the smaller thresholds, the \pm 0.02 diagrams in the lower half show more extremes of red and blue. Regardless of the model chosen, a positive region of the MEP clearly surrounds the $\mathrm{H}$ atom of HSF, while the F marks the most negative area. Several of the diagrams suggest a weaker negative region in the vicinity of a $\mathrm{S}$ lone pair. Based on this sort of analysis, one would anticipate that HSF would form its strongest interaction with a nucleophile like $\mathrm{NH}_{3}$ via its $\mathrm{H}$ atom, in a $\mathrm{SH} \cdot \mathrm{N} \mathrm{H}$ bonding arrangement. While such an arrangement does indeed represent a minimum on the potential energy surface, this conformation is only second in stability to a chalcogen bond where $\mathrm{NH}_{3}$ approaches the $\mathrm{S}$ atom opposite the $\mathrm{F}$ (see Fig. 1). The positive blue domain that would correspond to this region is most evident in the lower diagrams, using the \pm 0.02 au extrema. But none of these diagrams offer much guidance as to which of these two regions is more positive, and which might more strongly attract a nucleophile. The third structure appearing in the top half of Figure 1 also corresponds to a chalcogen bond, this time with the $\mathrm{N}$ opposite the $\mathrm{H}$ of HSF. Such a geometry is only hinted at by the MEPs in Figure 5 , only via a small blue region for $1.5 \times$ vdW, with extrema \pm 0.02 ; the other diagrams offer little real evidence of this geometry.

Many of the same observations apply to the other Lewis acid molecules considered here. For purposes of conciseness, Figure 6 illustrates the MEPs for all six molecules corresponding to $1.5 \mathrm{x}$ vdW radius and for MEP spanning the range $\pm 0.02 \mathrm{au}$, as this particular choice seems to offer the optimal means of predicting dimer geometries. The positive domains of $\mathrm{H}_{2} \mathrm{PF}$ and $\mathrm{H}_{2} \mathrm{PBr}$ would suggest either XP..N pnicogen bonding or $\mathrm{PH} \cdot \mathrm{N} \mathrm{H}$-bonds. While the former certainly occurs (see Fig. 2), and is the global minimum for each, it is only $\mathrm{PH}_{2} \mathrm{Br}$ that engages in a $\mathrm{HB}$, and a highly distorted one at that. The MEPs suggest neither the appearance of a HP..N pnicogen bond, which is reasonably strong nor even of the halogen bond for $\mathrm{PH}_{2} \mathrm{Br}$ (although the latter is rather weak, only 1.1 $\mathrm{kcal} / \mathrm{mol}$ ). With respect to the tetrels in the rightmost portion of Figure 6 , the only very blue region corresponds to the $\mathrm{XSi} \cdot \mathrm{N}$ tetrel bonds in Figure 3 . There is again little indication of neither the fairly strong $\mathrm{HSi} \cdot \cdot \mathrm{N}$ tetrel bonds nor the $\mathrm{SiBr} \cdot \mathrm{N}$ halogen bond, albeit a weak one.
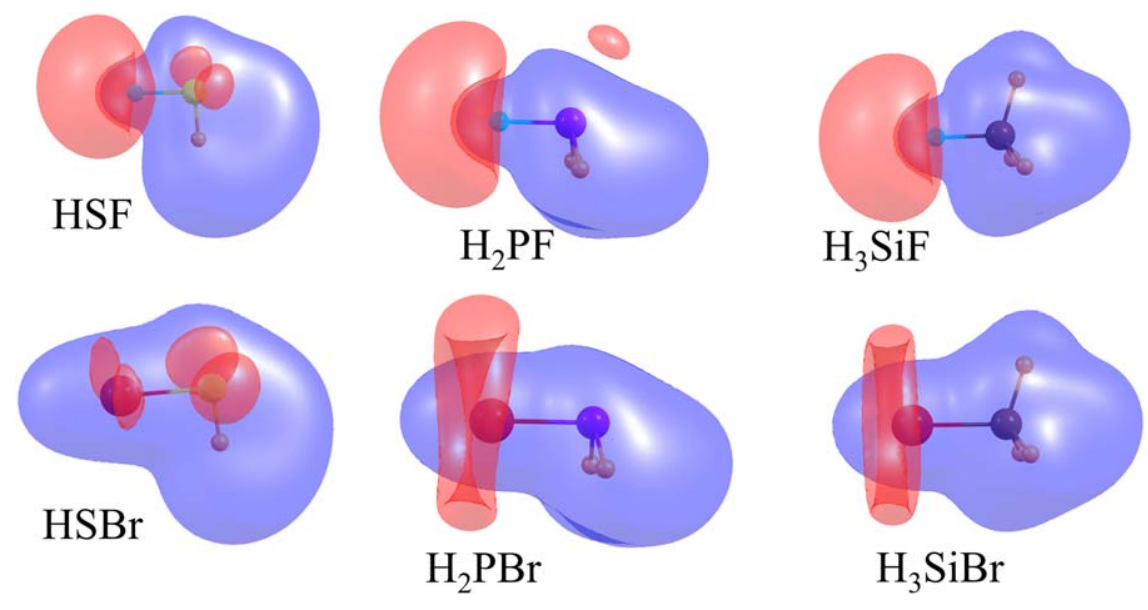

Figure 4. Molecular electrostatic potentials (MEPs) presented as an isopotential surface, for MEP $=+0.015$ au (blue) and -0.015 (red). [Color figure can be viewed at wileyonlinelibrary.com] 


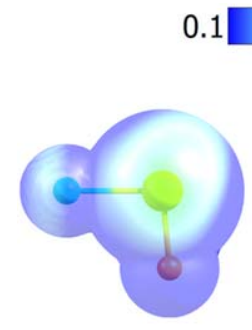

vdW

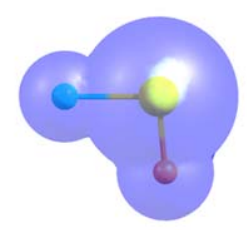

0.02
$-0.1$

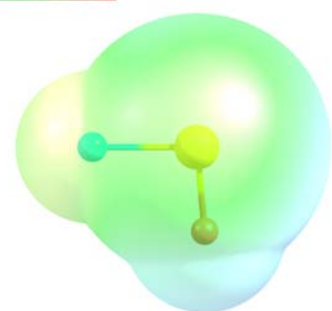

$1.5 \mathrm{x}$ vdW

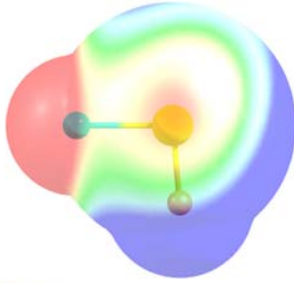

$-0.02$

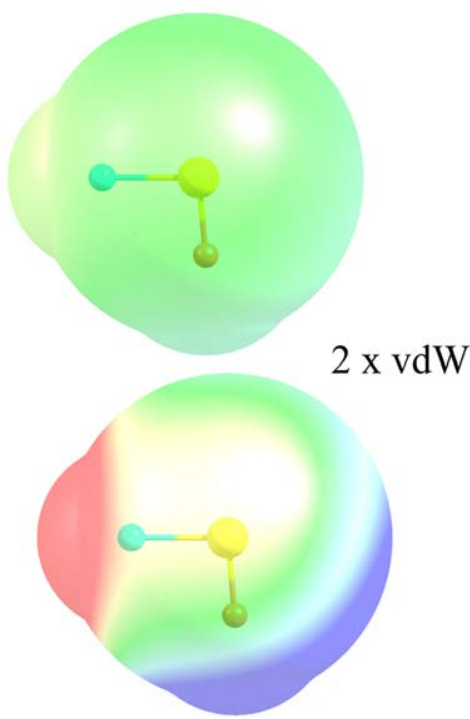

Figure 5. Molecular electrostatic potentials (MEPs) calculated for HSF on surfaces corresponding to various multiples of van der Waals radii, and showing indicated values of extrema, in au. [Color figure can be viewed at wileyonlinelibrary.com]

\section{MEP maximum point positions}

An alternate means of looking at MEPs is through the prism of specific points. More specifically, a surface is commonly chosen that surrounds the molecule of interest with a constant electron density. The extrema of the MEP are then identified on this isodensity surface. The specific location of each extremum can help to pinpoint the preferred direction for the approach of a partner molecule. Moreover, the numerical value of the MEP at these extrema can be compared with one another so as to assess which approach direction might be preferred. However, there are certain aspects of arbitrariness attached to this approach. In the first place, which isodensity surface offers the best window into the upcoming interaction? Large densities will place the surface under consideration rather close to the nuclei, while a smaller density choice will move this surface further out.

This sort of variation is illustrated in Figure 7 which shows the precise locations of the maximum, $V_{s, \max }$ on various isodensity surfaces for each of the various molecules serving as examples of Lewis acids. The small red dots refer to an isodensity of $0.01 \mathrm{au}$; black, green, and blue correspond to diminishing densities of $0.001,0.0002$, and 0.00005 au, respectively. As indicated above, these $V_{s, \max }$ markers move progressively further away from the molecule as the isodensity drops. But there are certain other interesting aspects of their positions. Taking HSF in the upper left of Figure 7 as an example, there are four locations where these maxima occur. There are maxima located on either end of both the $\mathrm{S}-\mathrm{H}$ and $\mathrm{S}-\mathrm{F}$ bonds. Those maxima on the $S$ ends correspond to the two sorts of
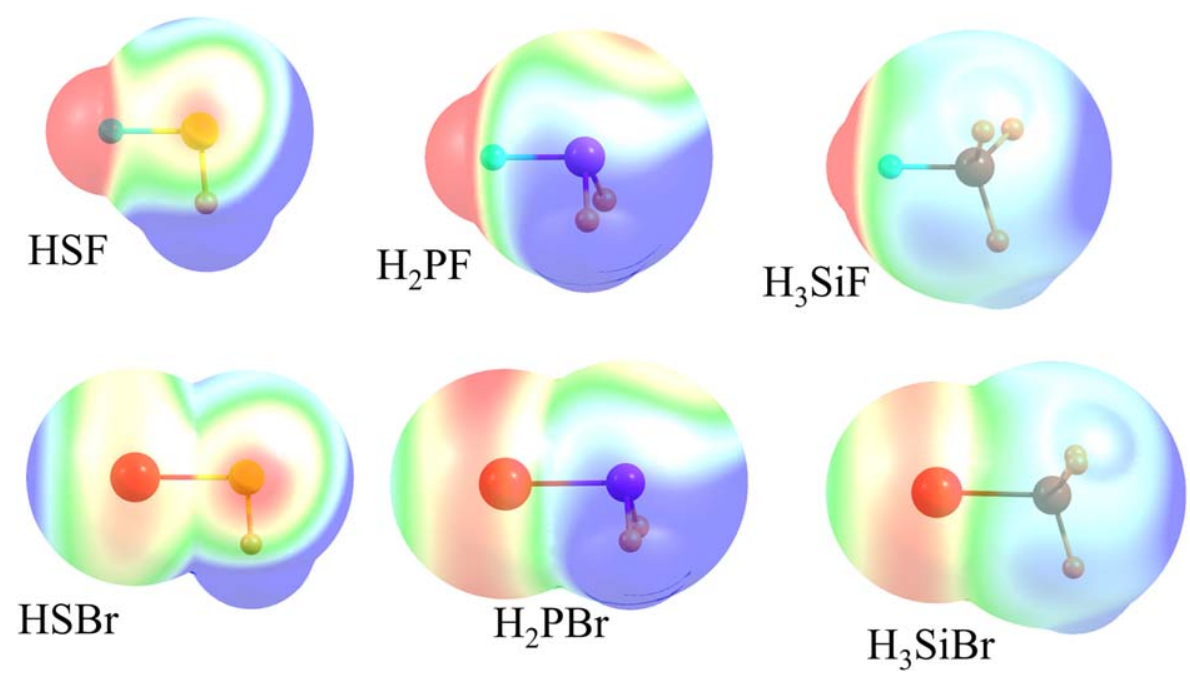

Figure 6. MEPs of indicated molecules, on surfaces corresponding to $1.5 \times$ van der Waals radii. Blue color indicates +0.02 au, and red shows -0.02 au. [Color figure can be viewed at wileyonlinelibrary.com] 


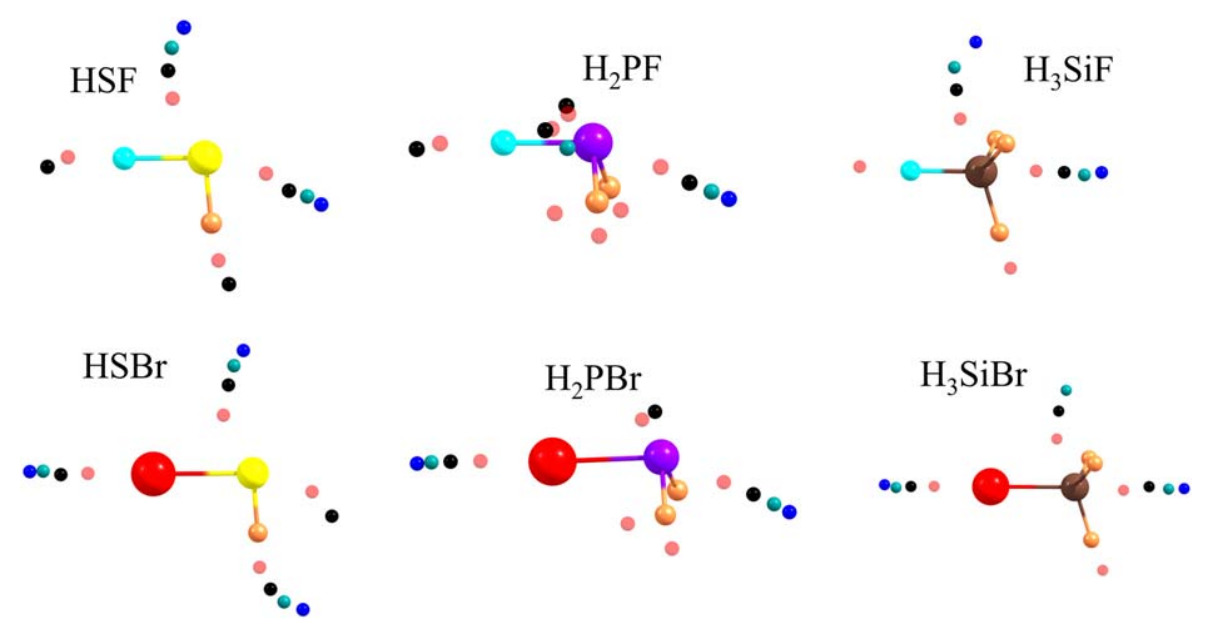

Figure 7. Points corresponding to $V_{s, \max }$ on surfaces corresponding to $\rho=0.01$ au (red), $\rho=0.001$ au (black), $\rho=0.0002$ au (green), and $\rho=0.00005$ au (blue). [Color figure can be viewed at wileyonlinelibrary.com]

chalcogen bonds highlighted in Figure 1. Maxima near the $\mathrm{H}$ are appropriate for the formation of a $\mathrm{SH} \cdot \cdot \mathrm{N} \mathrm{H}$-bond, while a possible halogen bond would take advantage of maxima near the $\mathrm{F}$ atom. Note, however, that despite the presence of the latter maxima, there is no minimum on the potential energy surface that encompasses a SF..N halogen bond. It is worth noting also that the maxima near the $\mathrm{S}$ and $\mathrm{H}$ atoms peter out as one moves further from the molecule. There are no green or blue dots characteristic of densities less than 0.001 au. The situation changes a bit for the $\mathrm{HSBr}$ molecule containing the larger halogen atom. In this case, there are four full maxima near both the $\mathrm{H}$ and $\mathrm{Br}$ atoms, but only two near the $\mathrm{S}$, along the Br-S axis.

The positions of the various $V_{\mathrm{s} \text {, max }}$ for the other Lewis acids are also displayed in Figure 7. Many of the patterns are repeated. There are four points corresponding to the pnicogen and tetrel bonds, opposite the halogen atom, consistent with structures of this type. There are also four points that represent a halogen bond for $\mathrm{Br}$, but fewer for $\mathrm{F}$, again consistent with $\mathrm{Br}$ halogen but not $\mathrm{F}$ halogen bonds. The tendency of any of these molecules to engage in a HB appears weak, with only a single point in the appropriate position, and only for the highest isodensity surface, $\rho=0.01$. This finding conforms to the rarity of $\mathrm{HB}$ structures. With respect to pnicogen or tetrel bonds opposite the $\mathrm{H}$ atom, this tendency is weak for $\mathrm{P}$ but much stronger for $\mathrm{Si}$ with more than two points in either case. Note that all Lewis acids form a dimer of this type, and that those with $\mathrm{Si}$ are indeed stronger than those involving $\mathrm{P}$.

With respect to the distances of these various points to their neighboring atoms, there is a good deal of variability. Taking the HSF molecule as an example, for $\rho=0.01 \mathrm{au}$, the points nearest the $S$ are 1.39 and $1.31 \AA$ from this atom. That near the $F$ is separated from it by $1.17 \AA$, and that near the $H$ is closer still at $0.78 \AA$. These distances all elongate as one moves to a smaller isodensity of $0.001 \mathrm{au}$, to $1.95,1.87,1.61$, and 1.31 $\AA$, respectively. When this density is reduced all the way down to $0.00005 \mathrm{au}$, the points move still further. The point near the $\mathrm{S}$, opposite the $\mathrm{F}$, that approximates the approach of the $\mathrm{NH}_{3}$ to form a FS. $\mathrm{N}$ chalcogen bond, is removed by $2.60 \AA$ from the $\mathrm{S}$.

How well do these various maxima predict the actual angle of approach of the nucleophile, with respect to its optimal orientation with the dimer? In some cases, the predicted angle of approach is largely independent of the isodensity surface chosen. Note that for HSF, for example, the four dots on the right side, opposite the $\mathrm{F}-\mathrm{S}$ bond are lined up in a nearly linear row. More exactly, the $\theta$ (FS.-max) angles are $165^{\circ}, 158^{\circ}, 159^{\circ}$, and $158^{\circ}$ respectively. These angles are all a bit less linear than the $171^{\circ}$ for the $\theta(\mathrm{FS} \cdot \mathrm{N})$ angle in the dimer in Figure 1. In other cases, the angle is rather dependent on the isodensity surface chosen. The four $\theta(\mathrm{HS} \cdot \cdot \mathrm{N})$ angles for the maxima lying opposite the $\mathrm{H}$ atom are $157^{\circ}, 163^{\circ}, 169^{\circ}$, and $177^{\circ}$, a span of $20^{\circ}$. The $\theta(\mathrm{HS} \cdot \mathrm{N})$ angle in the HS..N dimer in Figure 1 is $163^{\circ}$, but this may be artificially low, pulled away from linearity by a weak $\mathrm{NH} . \mathrm{F}$ attraction. (This same force may also be responsible for the deviation from linearity of the $\mathrm{H}$-bonded structure in Fig. 1.) Turning to the $\mathrm{HSBr}$ molecule, the $\mathrm{H}$-bonded structure is preferred, with a $\theta(\mathrm{SH} \cdot \cdot \mathrm{N})$ angle of $163^{\circ}$. (The $3.3 \AA$ distance from $\mathrm{Br}$ to $\mathrm{H}$ is probably too long to represent a significant $\mathrm{HB}$.) The positions of the four maxima near the $\mathrm{H}$ in $\mathrm{HSBr}$ do not reflect this disposition very well, with $\theta(\mathrm{SH} \cdot$.max) equal to $183^{\circ}, 188^{\circ}, 196^{\circ}$, and $205^{\circ}$. In other words, the maxima drift further and further away from the $\mathrm{Br}$ as the isodensity diminishes. Moreover, there are only two maxima near the $\mathrm{S}$ atom opposite the $\mathrm{Br}$, with no maxima for $\rho<0.001$. Yet the corresponding chalcogen bond is quite strong, at $4.7 \mathrm{kcal} / \mathrm{mol}$.

Examining Figure 7 as a whole leads to several generalities. The points opposite the halogen atom, whether $\mathrm{F}$ or $\mathrm{Br}$, fall nearly into a line, so the predicted angle of the ensuing chalcogen/pnicogen/tetrel bond is not dependent on choice of isodensity surface. The same may be said of the four points near the $\mathrm{Br}$ atom, all predicting a linear halogen bond. More dependent on choice of density is the angle opposite the $\mathrm{H}$ atom which arc away from the halogen as the isodensity diminishes, and one moves away from the molecule. The same may be said for the HB that might be formed by HSBr. 


\section{MEP maximum point values}

One can be more quantitative in this analysis by comparing the numerical values of the MEP at each of the maxima. First with respect to nomenclature, the maximum listed in Tables 1-3 lies closest to the second atom, directly opposite the first. For example, the X-S maximum in the first row of Table 1 lies along the X-S axis, nearest the $\mathrm{S}$. These quantities are listed in Tables 1-3 along with the computed binding energies. Of course, these $V_{s, \max }$ values decrease from left to right, as the isodensity used to define the surface diminishes, and one moves further away from the nuclei. But within the context of a given isodensity, there are some consistent trends. In the case of HSF in the upper half of Table 1, for example, the X-S maximum is consistently the largest in magnitude, followed by $\mathrm{S}-\mathrm{H}$, and then $\mathrm{H}-\mathrm{S}$. This pattern is consistent with the binding energies. Note, however, that the use of an isodensity smaller than $\rho=0.001$ would not predict a H-bonded structure, although this geometry is rather stable. In the case of $\mathrm{HSBr}$ in the lower half of Table 1 , the $\mathrm{X}-\mathrm{S}$ and $\mathrm{S}-\mathrm{H}$ maxima are very close in magnitude, followed then by $\mathrm{S}-\mathrm{X}$ and $\mathrm{H}-\mathrm{S}$ in that order. This pattern is fairly consistent with the binding energies, although again there is a problem with low densities where the X-S maximum disappears.

For the HSX molecules then, the values of $V_{s, \max }$ offer a fairly good predictive capability for the four possible minima on the potential energy surface. Their ordering conforms with the binding energies for the most part. However, there is a danger in using an isodensity that is too small, as certain important MEP maxima may disappear. There is also a tendency for the locations of these maxima to drift too far away from linearity, with respect to the actual geometries optimized for the dimers.

A similar sort of analysis of the pnicogen and tetrel bonding molecules leads to the MEP maxima in Tables 2 and 3, respectively. Again, one sees the lowering of the values of these maxima as one moves away from each molecule, as the isodensity contour drops to 0.00005 au. There is also the tendency of some of these maxima to disappear for the lower density surfaces. For example, the $\mathrm{H}-\mathrm{P}$ maximum for the $\mathrm{H}_{2} \mathrm{PX}$ molecules in Table 2 is only present for $\rho=0.01$ and $0.001 \mathrm{au}$. Note also that despite the appearance of a S-F or P-F maximum, $V_{s, \max }$ is actually negative for the highly electronegative $\mathrm{F}$ atom, consistent with the finding that a halogen-bonded structure does not represent a minimum for either HSF or $\mathrm{H}_{2} \mathrm{PF}$.

As a summation of the combined results in Tables (1-3), the most stable geometry of each molecule when paired with $\mathrm{NH}_{3}$ is a chalcogen/pnicogen/tetrel bonded structure, with the $\mathrm{N}$ directly opposite the halogen. The parallel situation, opposite one of the $\mathrm{H}$ atoms, is usually the second most stable, with the sole exception of the $\mathrm{SH} \cdot . \mathrm{N}$ HB for FSH. This energetic ordering is reflected in the pattern of $V_{s, \max }$ regardless of the particular isodensity chosen. Within a given sort of bond, for example, the pnicogen bonds in Table 2 , there is only modest correlation between the binding energy and $V_{\mathrm{s} \text {, max }}$. The latter correctly predicts the greater stability of the FP..N over the
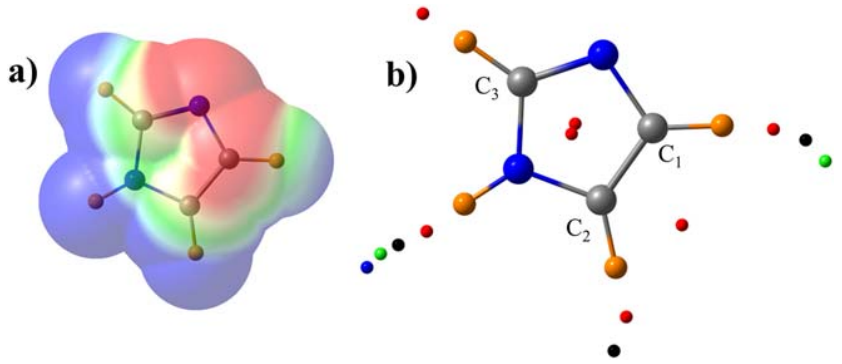

Figure 8. a) MEP of Im on surface corresponding to $1.5 \times$ van der Waals radii. Blue color indicates $+0.02 \mathrm{au}$, and red shows -0.02 au. b) $V_{\mathrm{s}, \max }$ points on isodensity surfaces corresponding to $\rho=0.01$ au (red), $\rho=0.001$ au (black), $\rho=0.0002$ au (green), and $\rho=0.00005$ au (blue). [Color figure can be viewed at wileyonlinelibrary.com]

BrP..N pnicogen bond, but not the equal stability of the HP..N bonds of the $\mathrm{F}$ and $\mathrm{Br}$ systems. Another curiosity involves the possible presence of a $\mathrm{PH} \cdot \mathrm{N}$ HB. The $\mathrm{H}_{2} \mathrm{PF}$ and $\mathrm{H}_{2} \mathrm{PBr}$ MEPs both have a maximum in this direction, and of a similar magnitude, but only for $\rho=0.01 \mathrm{au}$. Yet it is only for the latter molecule that such a minimum actually exists.

In terms of comparisons from one sort of bond to the next, one finds a decent correlation between binding energy and $V_{s, \max }$. Considering first the $\mathrm{Y} \cdot \mathrm{N}$ interactions in the top lines of Tables $1-3, E_{\mathrm{b}}$ follows the pattern:

$$
\mathrm{FS} \cdots \mathrm{N}>\mathrm{FP} \cdots \mathrm{N}>\mathrm{FSi} \cdot \mathrm{N}>\mathrm{BrSi} \cdot \mathrm{N}>\mathrm{BrP} \cdots \mathrm{N}>\mathrm{BrS} \cdot \mathrm{N}
$$

which compares with the $V_{\mathrm{s}, \max }$ order:

$\mathrm{FS} \cdot \mathrm{N}>\mathrm{BrSi} \cdot \mathrm{N}>\mathrm{FSi} \cdot \mathrm{N}>\mathrm{FP} \cdot \mathrm{N}>\mathrm{BrP} \cdot \mathrm{N}>\mathrm{BrS} \cdot \mathrm{N}$.

In other words, the latter quantity exaggerates the stability of the BrSi tetrel bond while underestimating the FP pnicogen bond.

\section{Aromatic system}

To broaden this discussion, the set of systems examined was expanded to include a heteroaromatic ring. The imidazole (Im) molecule is important in its own right, and also comprises the sidechain of the commonly occurring histidine residue of proteins. There are a number of regions of this molecule that could serve as Lewis acid sites. There is one $\mathrm{NH}$ proton donor group, combined with three different $\mathrm{CH}$ donors. In addition, there is the possibility that the area above the aromatic ring could attract an electrophile, via what is sometimes referred to as a $\pi$-hole.

The MEP of Im is displayed in Figure $8 a$, and the positions of its maxima on various isodensity surfaces in Figure $8 \mathrm{~b}$, the latter of which also contains the numbering used for the three $\mathrm{C}$ atoms. The MEP is rather positive (blue) around all four of the $\mathrm{H}$ atoms, and negative in the vicinity of the unprotonated $\mathrm{N}$ atom as well as above $\mathrm{C}_{1}$. The pattern of MEP maxima in Figure $8 \mathrm{~b}$ is rather interesting in a number of respects. These maxima appear along the extension of the $\mathrm{NH}$ bond for all four values of the potential, varying from 0.01 all the way down to $0.00005 \mathrm{au}$. There is only one such minimum along 
Table 4. Binding energies of $\mathrm{H}$-bonded complexes of imidazole with $\mathrm{NH}_{3}$ and $V_{s, \max }(\mathrm{kcal} / \mathrm{mol})$ on indicated isopotential surface of imidazole, $\rho$ in au.

$$
E_{\mathrm{b}}
$$

\begin{tabular}{lcrccc|}
$\mathrm{Im}$ & $(\mathrm{kcal} / \mathrm{mol})$ & $\rho=0.01$ & $\rho=0.001$ & $\rho=0.0002$ & $\rho=0.00005$ \\
\hline $\mathrm{NH}$ & 7.50 & 129.24 & 53.90 & 37.54 & 28.72 \\
$\mathrm{C}_{1} \mathrm{H}$ & 2.51 & 57.96 & 12.72 & 5.57 & $\mathrm{x}$ \\
$\mathrm{C}_{2} \mathrm{H}$ & $\mathrm{x}$ & 75.34 & 26.28 & $\mathrm{x}$ & $\mathrm{x}$ \\
$\mathrm{C}_{3} \mathrm{H}$ & $\mathrm{x}$ & 72.04 & $\mathrm{x}$ & $\mathrm{x}$ & $\mathrm{x}$ \\
$\mathrm{C}_{1}-\mathrm{C}_{2}$ & $\mathrm{x}$ & 38.28 & $\mathrm{x}$ & $\mathrm{x}$ & $\mathrm{x}$ \\
perp & $\mathrm{x}$ & 26.28 & $\mathrm{x}$ & $\mathrm{x}$ & $\mathrm{x}$ \\
\hline
\end{tabular}

$\mathrm{C}_{3} \mathrm{H}$, for the highest isodensity of $0.01 \mathrm{au} . \mathrm{C}_{2} \mathrm{H}$ has 2 such maxima and $\mathrm{C}_{1} \mathrm{H}$ three. From this perspective, one might anticipate that the order of $\mathrm{H}$-bonding strength to a proton acceptor ought to be $\mathrm{NH}>\mathrm{C}_{1} \mathrm{H}>\mathrm{C}_{2} \mathrm{H}>\mathrm{C}_{3} \mathrm{H}$. There are several other maxima, but only red ones, corresponding to the highest isodensity of $0.01 \mathrm{au}$. One such maximum occurs near the approximate $C_{1}-C_{2}$ bond midpoint. There are also a pair of maxima visible near the center of the Im ring, but which actually occur some $1.1 \AA$ above and below the ring plane.

A series of full geometry optimizations revealed that Im forms $\mathrm{HBs}$ to $\mathrm{NH}_{3}$ in only two modes, which is consistent with the pattern of maxima in Figure $8 \mathrm{~b}$. As indicated in Table 4, the $\mathrm{NH} \bullet \bullet \mathrm{N} \mathrm{H}$-bond amounts to $7.50 \mathrm{kcal} / \mathrm{mol}$, and the $\mathrm{C}_{1} \mathrm{H} \bullet \bullet \mathrm{N}$ bond to $2.51 \mathrm{kcal} / \mathrm{mol}$. None of the other maxima in Figure $8 \mathrm{~b}$ result in an optimized complex. This finding is not entirely consistent with the numerical values of these maxima. The $\mathrm{NH}$ site is clearly the most positive, affirming the strength of the $\mathrm{NH} \cdot . \mathrm{N} \mathrm{H}$-bond. Conversely, the $\mathrm{C}_{2} \mathrm{H}$ site is consistently more positive than $\mathrm{C}_{1} \mathrm{H}$, and by a substantial margin, even though the former group does not engage in a HB. Indeed, the same is true for $\mathrm{C}_{3} \mathrm{H}$. Thus if one were to predict the outcome based on $V_{s, \max }$ at either $\rho=0.01$ or $0.001 \mathrm{au}$, the incorrect guess of a $\mathrm{C}_{2} \mathrm{H} \cdot \cdot \mathrm{N}$ or $\mathrm{C}_{3} \mathrm{H} \cdot \mathrm{N} \mathrm{H}$-bond would have been made. Conversely, the small values of $V_{s, \max }$ for the $C_{1}-C_{2}$ midpoint or the points perpendicular to the Im plane, and their appearance only for $\rho=0.01$ au are consonant with the absence of corresponding minimum geometries.

\section{Summary and Discussion}

MEPs offer useful insights into the types of noncovalent bonding that can be expected on the approach of a pair of molecules. A scan of positive and negative regions provides reasonable guesses as to how the two molecules might orient themselves within a heterodimer. The usefulness of these pictorial representations is compromised if the isopotential surface scanned is of too large a magnitude, as this might miss negative regions entirely. Too small potential risks looking at regions too far away from the molecule of interest. Similar restrictions apply to maps corresponding to particular distances from the nuclei. Consideration of areas that lie too close to the nuclei, on van der Waals surfaces, is of little utility as the MEP is largely positive over the entire region. But considering surfaces that are more removed, at 1.5-2.0 times the vdW radii, allows a more nuanced and helpful view of the variation in sign and magnitude of the MEP. Conversely, these pictures are unable to predict precise angles of approach for the two molecules, as the positive regions are broad in area, and may encompass two or more different regions of a particular molecule. Another weakness lies in their inability to quantitatively compare the energetics of various different sorts of noncovalent bonds, for example, $\mathrm{H}$ versus chalcogen bonds. Moreover, these pictorial MEPs can fail to predict certain structures that have competitive stabilities with those that are more obvious from these diagrams.

The identification of points where the MEP reaches a maximum allow for a more quantitative assessment of various regions, and offers a means of predicting in advance which sort of interaction might prove to be most stable. Again, there is a certain amount of arbitrariness in terms of which particular surface is analyzed; an isodensity of 0.001 au is commonly used but is by no means the only choice. Smaller isodensities of course move the $V_{s, \max }$ points out further away from the nuclei. In some cases, this outward displacement has little to no effect on their angular position. For example, the choice of $\rho$ does not affect the prediction as to the angles involved in the $\mathrm{F}-\mathrm{X} \cdot \mathrm{N}$ chalcogen, pnicogen, or tetrel bonds, or the $\mathrm{X}-\mathrm{Br} \cdot \mathrm{N}$ halogen bonds. The same cannot be said, however, concerning these same bonds when the nucleophile is positioned opposite the $\mathrm{H}$ atom rather than $\mathrm{F}$. The predicted $\mathrm{SH} \cdot . \mathrm{N} \mathrm{H}$-bond angle, too, would vary a good deal depending on the particular choice of $\rho$. Another issue is the observation that some of these points disappear as $\rho$ diminishes. For example, there are maxima near the F atom in FSH for $\rho=0.01$ and $0.001 \mathrm{au}$, but no such maxima for smaller $\rho$. The former values might then lead to the erroneous conclusion that a SF..N halogen bond might be formed, whereas the latter prediction of no such configuration would be correct. The opposite can also occur. As an example, there is a maximum near the $\mathrm{H}$ atoms bonded to Si for the highest density $\rho=0.01 \mathrm{au}$, but no $\mathrm{H}$-bonded minimum on the potential energy surface.

The numerical values of $V_{s, \max }$ permit a quantitative comparison of the stabilities of the various sorts of bonding for a given pair of molecules. With some exceptions, these predictions are largely borne out. Taking $\mathrm{H}_{2} \mathrm{PBr}$ as an example, the $V_{\mathrm{s}, \max }$ trend, for $\rho=0.01$ au, of $\mathrm{BrP} \cdot . \mathrm{N}>\mathrm{HP} \cdot \mathrm{N}>\mathrm{PH} \cdot \mathrm{N}>\mathrm{PBr} \cdot \mathrm{N}$ precisely matches the energetic ordering of these heterodimer arrangements. This close correlation begins to fall apart, however, for smaller isodensities, where there are no maxima located near the $\mathrm{H}$ atom. The data presented here would thus argue for the use of the larger isodensity of 0.01 au which avoids the loss of maxima that begin to occur for $0.001 \mathrm{au}$, for configurations that do indeed represent minima on the potential energy surface. Conversely, the failure to identify certain MEP maxima by $\rho=0.001$ au is not necessarily pejorative, as this isodensity avoids some of the "false positives" associated with the larger density where a MEP maximum does not correspond to an equilibrium geometry. Going beyond $\rho=0.001$ au to smaller isodensities is probably best avoided as too many MEP maxima are missed, many of which correspond to equilibrium geometries.

In the context of imidazole, there are a number of regions where a search of an isodensity surface reveals a maximum, 
but not all of them result in an equilibrium structure. Maxima are observed near to each of the four protons, above and below the imidazole plane, and directly between two $\mathrm{C}$ atoms. However, it is only the $\mathrm{NH}$ and one of the three $\mathrm{CH}$ groups that engage in a $\mathrm{HB}$ with $\mathrm{NH}_{3}$. But comparison of the numerical values of $V_{s, \max }$ would lead to an erroneous prediction as to which of the $\mathrm{CH}$ groups are most amenable to a $\mathrm{HB}$.

In summary then, examination of the MEP does indeed provide useful information in many cases. However, there are certain issues which are imperfectly addressed without due consideration of other factors. It is important to recall that the data presented here focuses on only one component of each interaction, the electrostatic attraction between the static electron densities, prior to any polarization or charge transfer effects. A good deal of work has documented that while this component is indeed important, there are other aspects of the interaction that cannot be ignored. There are in particular second-order electron density perturbations, which are commonly referred to as polarization and/or charge transfer. This component is augmented by London dispersion, another attractive term, which can be quite appreciable in magnitude. While dispersion tends to be rather isotropic, charge transfer is heavily anisotropic. It is partly responsible for the trend toward linearity of hydrogen, pnicogen, halogen bonds, and so forth. This angular preference can be understood on the basis of charge transfer from a lone pair of the Lewis base to the $\sigma^{*}$ antibonding orbital within the Lewis acid, which is maximized by the proper alignment of these orbitals. It is indeed this same transfer which has been deemed responsible for the red shift of the $\mathrm{X}-\mathrm{H}$ stretching frequency in the proton donor within a HB. And lastly, steric repulsive forces have also been shown ${ }^{[61-63]}$ to have a large effect on the angular features of hydrogen and other noncovalent bonds. It would thus be a mistake to assume that the angular features of any of the noncovalent bonds examined here are controlled exclusively by electrostatics. It is, therefore, not entirely surprising that the intermolecular angles within the equilibrium geometries can deviate significantly from those predicted purely on the basis of the positions of the MEP maxima.

Keywords: molecular electrostatic potential • isodensity surface • isopotential · chalcogen bond $\cdot$ tetrel bond $\cdot$ pnicogen bond

How to cite this article: S. Scheiner. J. Comput. Chem. 2018, 39 500-510. DOI: $10.1002 /$ jcc.25085

\section{References}

[1] K. Müller-Dethlefs, P. Hobza, Chem. Rev. 2000, 100, 143

[2] K. E. Riley, M. Pitok, P. Jureka, P. Hobza, Chem. Rev. 2010, 110, 5023.

[3] S. Scheiner, Ed. Noncovalent Forces; Springer: Heidelberg, 2015.

[4] G. C. Pimentel, A. L. McClellan, The Hydrogen Bond; Freeman: San Francisco, 1960.

[5] M. L. Huggins, Angew. Chem., Int. Ed. Engl. 1971, 10, 147.

[6] M. D. Joesten, L. J. Schaad, Hydrogen Bonding; Marcel Dekker: New York, 1974.
[7] P. Schuster, G. Zundel, C. Sandorfy, Eds. The Hydrogen Bond. Recent Developments in Theory and Experiments; North-Holland Publishing Co.: Amsterdam, 1976.

[8] S. Scheiner, Hydrogen Bonding. A Theoretical Perspective; Oxford University Press: New York, 1997.

[9] P. Politzer, D. G. Truhlar, Eds. Chemical Applications of Atomic and Molecular Electrostatic Potentials; Plenum: New York, 1981.

[10] J. Tomasi, B. Mennuci, M. Cammy, Molecular Electrostatic Potentials: Concepts and Applications; Elsevier: Amsterdam, 1996.

[11] K. Kitaura, K. Morokuma, Int. J. Quantum Chem. 1976, 10, 325.

[12] R. Cammi, F. J. O. Del Valle, J. Tomasi, Chem. Phys. 1988, 122, 63.

[13] S. M. Cybulski, S. Scheiner, Chem. Phys. Lett. 1990, 166, 57.

[14] E. D. Glendening, A. Streitwieser, J. Chem. Phys. 1994, 100, 2900.

[15] S. Scheiner, J. Phys. Chem. B 2005, 109, 16132.

[16] K. U. Lao, J. M. Herbert, J. Chem. Theory Comput. 2016, 12, 2569.

[17] S. Scheiner, L. Wang, J. Am. Chem. Soc. 1993, 115, 1958.

[18] T. A. Schaub, R. Sure, F. Hampel, S. Grimme, M. Kivala, Chem. Eur. J. 2017, 23, 5687.

[19] P. Politzer, J. S. Murray, Theor. Chem. Acc. 2012, 131, 1114.

[20] J. Rezac, A. de la Lande, Phys. Chem. Chem. Phys. 2017, 19, 791.

[21] I. Alkorta, J. Elguero, J. E. Del Bene, ChemPhysChem 2016, 17, 3112.

[22] B. Nepal, S. Scheiner, Phys. Chem. Chem. Phys. 2016, 18, 18015.

[23] M. D. Esrafili, H. Akhgarpour, Mol. Phys. 2016, 114, 1847.

[24] V. d P N. Nziko, S. Scheiner, J. Phys. Chem. A 2015, 119, 5889.

[25] D. Quiñonero, Molecules 2015, 20, 11632.

[26] S. Scheiner, U. Adhikari, J. Phys. Chem. A 2011, 115, 11101.

[27] A. Bauzá, T. J. Mooibroek, A. Frontera, ChemPhysChem 2016, 17, 1608.

[28] I. Alkorta, J. Del Bene, J. Elguero, Crystals 2016, 6, 19

[29] Q. Li, H. Zhuo, X. Yang, J. Cheng, W. Li, R. E. Loffredo, ChemPhysChem 2014, 15, 500.

[30] S. Scheiner, Int. J. Quantum Chem. 2013, 113, 1609.

[31] A. Bauzá, D. Quiñonero, P. M. Deyà, A. Frontera, CrystEngComm 2013, $15,3137$.

[32] S. Scheiner, Chem. Eur. J. 2016, 22, 18850

[33] M. D. Esrafili, M. Vakili, M. Javaheri, H. R. Sobhi, Mol. Phys. 2016, 114, 1974.

[34] V. d P N. Nziko, S. Scheiner, Phys. Chem. Chem. Phys. 2016, 18, 3581.

[35] S. Scheiner, Chem. Phys. Lett. 2011, 514, 32.

[36] S. J. Grabowski, Phys. Chem. Chem. Phys. 2014, 16, 1824.

[37] S. R. Gadre, R. K. Pathak, Proc. Indian Acad. Sci. (Chem. Sci.) 1990, 102, 189.

[38] S. R. Gadre, I. H. Shrivastava, J. Chem. Phys. 1991, 94, 4384.

[39] S. R. Gadre, P. K. Bhadane, J. Chem. Phys. 1997, 107, 5625.

[40] A. Kumar, S. R. Gadre, N. Mohan, C. H. Suresh, J. Phys. Chem. A 2014, $118,526$.

[41] J. S. Murray, P. Politzer, J. Org. Chem. 1991, 56, 6715.

[42] H. Hagelin, J. S. Murray, T. Brinck, M. Berthelot, P. Politzer, Can. J. Chem. 1995, 73, 483.

[43] P. Politzer, J. S. Murray, M. C. Concha, J. Mol. Model. 2007, 13, 643.

[44] P. Politzer, J. S. Murray, Z. Peralta-Inga, Int. J. Quantum Chem. 2001, 85, 676.

[45] T. Clark, M. Hennemann, J. S. Murray, P. Politzer, J. Mol. Model. 2007, 13, 291.

[46] J. S. Murray, P. Lane, P. Politzer, J. Mol. Model. 2009, 15, 723.

[47] E. D. Stevens, Mol. Phys. 1979, 37, 27.

[48] J. Tomasi, In Chemical Applications of Atomic and Molecular Electrostatic Potentials; P. Politzer, D. G. Truhlar, Eds.; Plenum: New York, 1981; p. 257.

[49] S. Ikuta, J. Mol. Struct. (Theochem) 1990, 205, 191.

[50] J. P. M. Lommerse, A. J. Stone, R. Taylor, F. H. Allen, J. Am. Chem. Soc. 1996, 118, 3108

[51] I. Alkorta, S. Rozas, J. Elguero, J. Phys. Chem. A 1998, 102, 9278.

[52] R. M. Minyaev, V. I. Minkin, Can. J. Chem. 1998, 76, 776.

[53] W. Scherer, M. Spiegler, B. Pedersen, M. Tafipolsky, W. Hieringer, B. Reinhard, A. J. Downs, G. S. McGrady, Chem. Commun. 2000, 2000, 635.

[54] S. Jenkins, I. Morrison, Chem. Phys. Lett. 2000, 317, 97.

[55] H. Torii, J. Chem. Phys. 2003, 119, 2192.

[56] S. J. Grabowski, E. Bilewicz, Chem. Phys. Lett. 2006, 427, 51.

[57] M. J. Frisch, G. W. Trucks, H. B. Schlegel, G. E. Scuseria, M. A. Robb, J. R. Cheeseman, G. Scalmani, V. Barone, B. Mennucci, G. A. Petersson, H. Nakatsuji, M. Caricato, X. Li, H. P. Hratchian, A. F. Izmaylov, J. Bloino, G. Zheng, J. L. Sonnenberg, M. Hada, M. Ehara, K. Toyota, R. Fukuda, J. 
Hasegawa, M. Ishida, T. Nakajima, Y. Honda, O. Kitao, H. Nakai, T. Vreven, J. A. Montgomery, Jr., J. E. Peralta, F. Ogliaro, M. Bearpark, J. J. Heyd, E. Brothers, K. N. Kudin, V. N. Staroverov, R. Kobayashi, J. Normand, K. Raghavachari, A. Rendell, J. C. Burant, S. S. lyengar, J. Tomasi, M. Cossi, N. Rega, J. M. Millam, M. Klene, J. E. Knox, J. B. Cross, V. Bakken, C. Adamo, J. Jaramillo, R. Gomperts, R. E. Stratmann, O. Yazyev, A. J. Austin, R. Cammi, C. Pomelli, J. W. Ochterski, R. L. Martin K. Morokuma, V. G. Zakrzewski, G. A. Voth, P. Salvador, J. J. Dannenberg, S. Dapprich, A. D. Daniels, O. Farkas, J. B. Foresman, J. V. Ortiz, J. Cioslowski, D. J. Fox, Revision B.01, Gaussian, Inc., Wallingford, CT.

[58] S. F. Boys, F. Bernardi, Mol. Phys. 1970, 19, 553.
[59] G. A. Zhurko, Chemcraft. Available at: http://www.Chemcraftprog.Com, accessed on October 09, 2017.

[60] T. Lu, F. Chen, J. Comput. Chem. 2012, 33, 580.

[61] M. Palusiak, J. Mol. Struct. (Theochem) 2010, 945, 89.

[62] U. Adhikari, S. Scheiner, Chem. Phys. Lett. 2012, 532, 31.

[63] A. J. Stone, J. Am. Chem. Soc. 2013, 135, 7005.

Received: 4 May 2017

Revised: 22 September 2017

Accepted: 29 September 2017

Published online on 30 October 2017 